\title{
Design and Implementation of a Digital Bracelet to Regulate the Rhythm of the Heart
}

\author{
Urbina Avila Gustavo Amiel \\ Colín Jiménez Silvia Guadalupe \\ Gutiérrez, Herrera Pablo \\ Marrón Noé Domingo \\ Caballero Alfaro Arístides \\ Mendoza Montero Fátima $Y$. \\ Avila Pérez Tagle Alfonso
}

Tecnológico Nacional de México Instituto Tecnológico de Milpa Alta San

Salvador Cuauhtenco, Delegación Milpa Alta, Ciudad de México, México

Doi: 10.19044/esj.2018.c3p15 $\quad$ URL:http://dx.doi.org/10.19044/esj.2018.c3p15

\begin{abstract}
The aim of this present study is to help people suffering from cardiovascular diseases by designing and implementing a digital bracelet that allows keeping a record of cardiac behavior to generate a more optimal diagnosis of the patient's current state and the evolution of the heart rate of the same patient. The research used the evolutionary methodology. This methodology consisted of three stages: specification, development, and validation. In this study, 95\% reliability was used. The results obtained with the present study were favorable. In addition, the digital bracelet has an important acceptance both in the patients and in the doctors who attended the people with cardiovascular disease.
\end{abstract}

Keywords: Cardiovascular diseases, digital bracelet, cardiac behavior

\section{Introduction}

Epidemiological research on the causes of cardiovascular diseases (CVD) began at the beginning of the century. The influence of diet on the development of arteriosclerosis was first described in 1913 by Anitschkow. The incidence of CVD increased from the beginning of the century to the point where it became the leading cause of death in industrialized countries. However, this was because each country changed its traditional lifestyles. In the United States, this happened in 1920; in Spain, it did not happen until the end of the decade of the 50 (UNED, 2017). 
The World Health Organization (WHO) (2013) argues that life expectancy at birth has increased substantially in recent decades worldwide. Concurring with the same ideas of the World Health Organization (2013), this organization has registered that it has gone from 64 years on an average in 1990 (62 in men and 67 in women) to 70 years in 2011 (68 in men and 72 in women).

In regards to the 34 states that make up the Organization for Economic Cooperation and Development (OECD), an average of 80.1 years was reached in 2011. This average represents an increase of 10 years compared to the 1970 data.

Spain was more than two years above that average with 82.4 years and is only below Switzerland (82.8 years), Japan (82.7 years), and Italy (82.7 years) (OECD, 2013).

\section{Cardiovascular Diseases}

Barrera (2015) argues that cardiovascular diseases are a group of disorders of the heart and blood vessels. The term "cardiovascular diseases" is used to group different types of diseases related to the heart or blood vessels (arteries and veins). The said expression describes any condition that compromises the cardiovascular system. Also, it is commonly used to refer to atherosclerosis. These pathologies have causes, mechanisms, and related treatments.

The World Health Organization (2015) mentions that cardiovascular diseases (CVD) are a group of disorders of the heart and blood vessels, including:

- $\quad$ Coronary heart disease: disease of the blood vessels that supply the heart muscle;

- Cerebrovascular diseases: diseases of the blood vessels that supply the brain;

- $\quad$ Peripheral arteriopathies: diseases of the blood vessels that supply the upper and lower limbs;

- $\quad$ Rheumatic heart disease: injuries of the heart muscle and heart valves due to rheumatic fever, a disease caused by bacteria called streptococci;

- Congenital heart disease: malformations of the heart present from birth;

- Deep vein thromboses and pulmonary embolisms: blood clots (thrombi) in the veins of the legs, which can detach (emboli) and lodge in the vessels of the heart and lungs.

Likewise, the World Health Organization (2015) argues that heart attacks and strokes are usually acute phenomena that mainly occur due to obstructions that prevent blood from flowing to the heart or brain. The most frequent cause is the formation of deposits of fat in the walls of the blood 
vessels that supply the heart or brain. CVA may also be due to hemorrhages of cerebral vessels or blood clots. Heart attacks and strokes are often caused by the presence of a combination of risk factors, such as smoking, unhealthy diets and obesity, physical inactivity, harmful alcohol consumption, high blood pressure, diabetes, and hyperlipidemia.

\section{Cardiovascular Diseases Worldwide}

According to the data of the World Health Organization (2015):

- CVDs are the leading cause of death worldwide. Every year, more people die from CVD than from any other cause.

- It is estimated that 17.5 million people died in 2012, representing $31 \%$ of all deaths worldwide. Of these deaths, 7.4 million were due to coronary heart disease, and 6.7 million due to stroke.

- More than three quarters of deaths due to CVD occur in low and middle income countries.

- Of the 16 million deaths of people under 70 years of age attributable to non-communicable diseases, $82 \%$ correspond to low and middle income countries and $37 \%$ are due to CVD.

- Most CVD can be prevented by acting on behavioral risk factors, such as tobacco use, unhealthy diets and obesity, physical inactivity or harmful alcohol consumption, and using strategies that cover the entire population.

- For people with CVD or with high cardiovascular risk (due to the presence of one or more risk factors, such as high blood pressure, diabetes, hyperlipidemia or some CVD already confirmed), early detection and early treatment are essential. This can be done through counseling services or drug administration as appropriate.

\section{Main Risk Factors}

Association (2017) mentions that the most important causes of heart disease and stroke are an unhealthy diet, physical inactivity, tobacco consumption, and the harmful consumption of alcohol. The effects of behavioral risk factors can manifest in people in the form of high blood pressure, hyperglycemia, hyperlipidemia, and overweight or obesity. These intermediate risk factors, which can be measured in primary care centers, are indicative of an increased risk of heart attacks, strokes, heart failure, and other complications.

\section{Common Symptoms of Cardiovascular Diseases}

Association (2017) mentions that the underlying disease of the blood vessels often does not present symptoms, and its first manifestation can be a heart attack or a stroke. The symptoms of heart attack consist of: 
- $\quad$ Pain or discomfort in the chest,

- $\quad$ Pain or discomfort in the arms, left shoulder, jaw or back.

In addition, there may be difficulty breathing, nausea or vomiting, dizziness or fainting, cold sweats and paleness. Shortness of breath, nausea and vomiting, and pain in the jaw or back are more frequent in women. Continuing with the same ideas of Association (2017), the most common symptom of CVA is the sudden, usually unilateral, loss of muscle strength in the arms, legs or face. Other symptoms consist of:

- The sudden onset, usually unilateral, of numbness in the face, legs, or arms;

- Confusion, difficulty speaking or understanding what is said;

- Visual problems in one or both eyes;

- Difficulty walking, dizziness, loss of balance or coordination;

- Severe headache of unknown cause;

- Weakness or loss of consciousness.

\section{Cardiovascular Diseases in Mexico}

According to data from the World Health Organization (2014), he argues that cardiovascular diseases are responsible for 54 percent of deaths that occur every year in Mexico, and some of them are due to lack of adherence to treatment .

According to the National Institute of Public Health (INSP), 68.5 percent of Mexicans have problems of obesity and overweight; 58 percent have diabetes; $21 \%$ has dyslipidemia; and another $43.2 \%$ hypertension. These conditions compared to the last 13 years have grown by 22 percent. Around the world, at least one in three people lose their lives due to a pathology related to cardiovascular diseases. This was according to statistics disseminated here by the INSP and the Mexican Society of Cardiology.

\section{Evolutionary Theory}

Ortega (2013) points out that the evolutionary methodology was proposed by Mills in 1980. He suggested the incremental approach to development as a way to reduce the repetition of work in the development process and give an opportunity to delay decision-making based on the requirements until it acquires experience with the system.

Evolutionary development is a software development methodology closely related to, but clearly distinct from, development by prototypes. Therefore, emphasis is placed on the importance of obtaining a flexible and expandable production system. Thus, if the requirements change during the development of the system, then a flexible work system can be developed with a minimum effort and time.

The fundamental difference between evolutionary development and software prototypes is that evolutionary development seeks to replace the old 
system with a new one that would have the property of satisfying new requirements as quickly as possible. In contrast, prototypes use an iterative approach only to determine generational requirements. Therefore, the time taken between each Hite ration is much more important for the evolutionary development. Also, the stages of evolutionary development aim to extend the increments of an operational software product in the directions determined by the evolution of the operational experience.

The model of evolutionary development can be ideally associated with a fourth generation application language and better still situations in which the user says: I cannot talk to you about what I want, but I would recognize it if I saw it. Thus, this method would quickly provide the user with an initial operational capacity and, in addition, establish a real operating basis to determine the subsequent improvements in the product.

With the existence of the evolutionary method, a new problem in the development of systems is configured. That is, the crisis now expands in the sense that not only is it required to reflect as accurately as possible the needs of the user, but now the environments in which the system is inserted are subject to change. As a result, these changes affect the effectiveness of the software that was developed. This was articulated by Lehman at the beginning of the eighties, when defining the laws of software evolution, whereby the first two laws are directly related to what is described.

Lehman proposes that the evolution of a software system is subject to several laws. It has determined these laws from experimental observations of several systems, such as large operating systems (...). Lehman says that there are five laws of the evolution of the programs:

- $\quad$ Continuous Change.

- A program that is used in a real-world environment must change or become less and less useful in that environment.

- Increasing complexity.

- As an evolving program changes, its structure becomes more complex, unless active efforts are made to avoid this phenomenon.

- $\quad$ Evolution of the program.

- The evolution of the program is a regulatory authoring process and a measurement of system attributes, such as size, time between versions, and number of errors noticed, etc. Thus, this reveals significant statistical trends and invariant characteristics.

- $\quad$ Conservation of organizational stability.

- During the life of a program, its speed of development is almost constant and independent of the resources dedicated to the development of the system.

- Conservation of familiarity. 


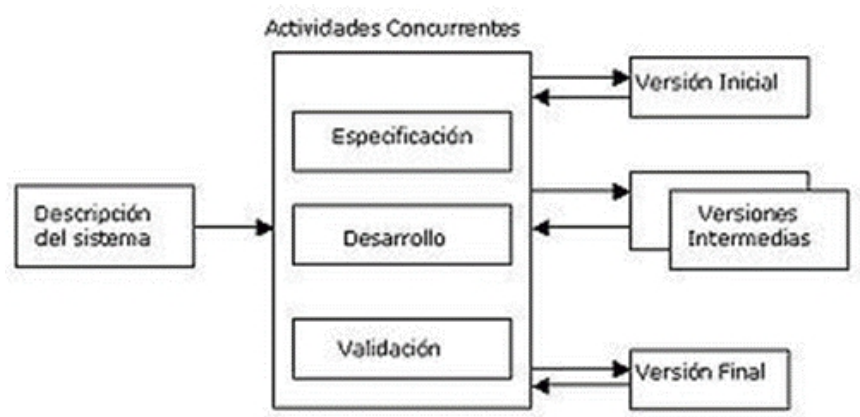

Figure 1. Example of the evolutionary methodology

\section{General Objective}

heart

Design and implement a digital wristband to regulate the rhythm of the

\section{Justification}

In more than $60 \%$ of cases, these risk factors are not adequately controlled, and improvements in this field remain scarce. More than one-third of patients with acute myocardial infarction die before reaching the hospital without receiving effective treatment (Banegas, Villar, Graciani \& Rodríguez, 2006).

Eguizábal (2014) argues that mobile health applications generated in 2015 is a business of 4,000 million euros in Spain. They are the third fastest growing category, behind games and utilities.

In Mexico, there is no bracelet that helps regulate heart rhythm in patients suffering from cardiovascular disease.

\section{Methodology}

The evolutionary methodology was chosen as a result of a consensus with all the members of the team and those methodologies that had more voting. Also, the choice of the team was also because the methodology admits a greater margin of error, for example, a specific cascade methodology that can only be advanced if the previous stages have been completed. This is an inconvenience since in advancing, you may encounter problems such as: poorly stated requirements, a poor system finish, unforeseen errors, etc.

\section{Initial Specification}

According to data from INEGI (2015), Mexico has 8.9 million inhabitants of which $30 \%$ of its population suffers from heart problems. Mexico ranks second for this cause. That is why the Digital visa company emerged. 
The digital wristband records heart beats per minute. By means of a heart rate sensor, it also allows entering personal and clinical data, as well as selecting the type of muscular pain that the patient has at the moment (left shoulder, head, chest, thoracic). Pain makes the time of beginning and ending to be stored in a database, specifying the time it was given and when it ended.

This bracelet has:

o Oled touch screen. ${ }^{\circledR}$

o Plate raspberry pi 3 MODEL B. ${ }^{\circledR}$

o Heart rate sensor. ${ }^{\circledR}$

o Rechargeable battery. ${ }^{\circledR}$

o Memory micro sd class 10 . ${ }^{\circledR}$

\section{Development}

The research started with the prototype, which is characterized by the design of the interface. Figure 2 shows an example of prototype 1.

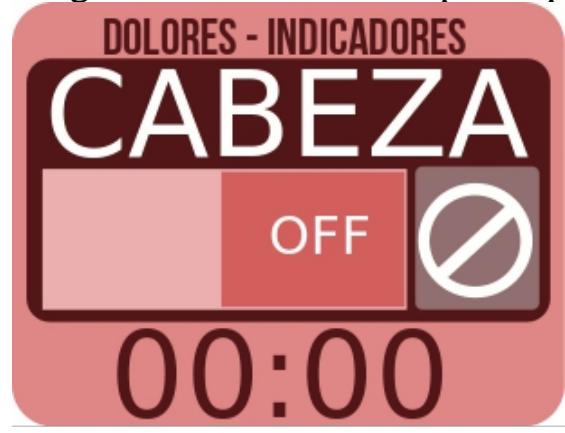

Figure 2. Example of prototype 1

After the interface continues to improve, Figure 3 shows an example of prototype 2 .

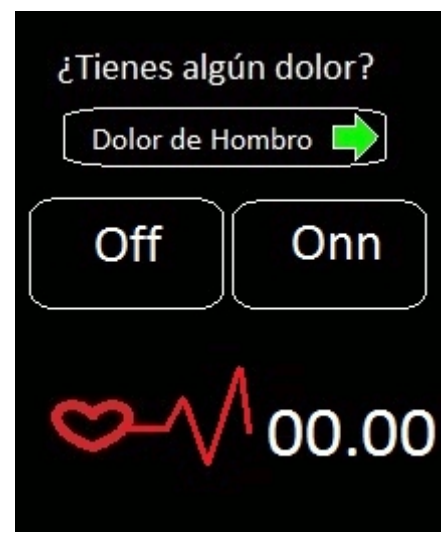

Figure 3. Example of prototype 2 
As the investigation progressed, the interface was restructured. However, Figure 4 shows an example of the prototype 3; likewise the following was added:

- $\quad$ Start (will serve to start running the pain time).

- $\quad$ Stop (for pain).

- $\quad$ Send (send the data collected to a database).

- $\quad$ Counter (which will let the specialist know how long the muscle pain lasted).

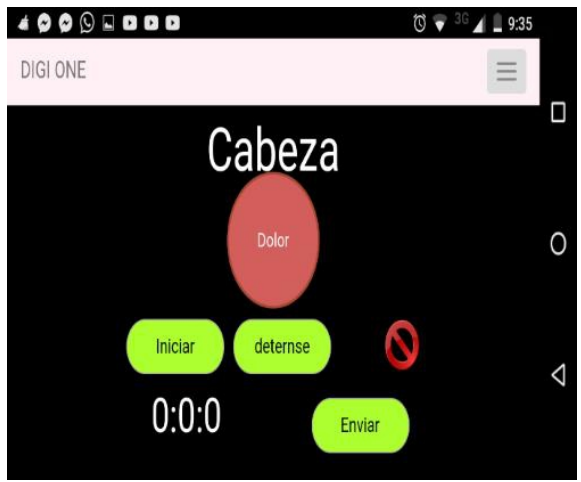

Figure 4. Example of prototype 3

The investigation proceeded with acceptable results. Figure 5 shows the idea (prototype 4) of the design of the bracelet.

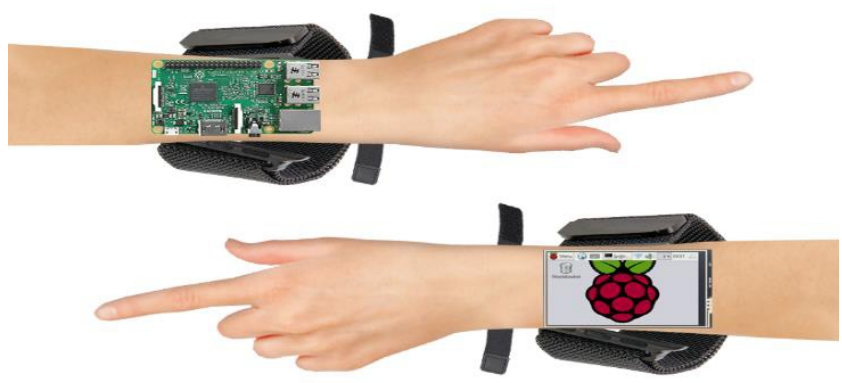

Figure 5. Example of prototype 4

The investigation continued to obtain positive results. Figure 6 (prototype 5) shows the final interface. 


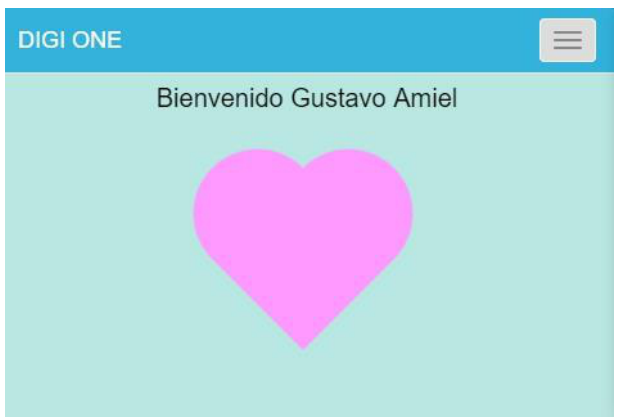

Figure 6. Example of prototype 5

In the programming part, the most sophisticated in the technology industry was used. In the case of the database, firebase was used. It is a nonrelational database of documents and the information is handled perfectly in real time. It is compatible with mobile devices and web services. This information about the database was taken from Tamplin and Lee (2011). Figure 7 shows part of the code generated through the investigation.

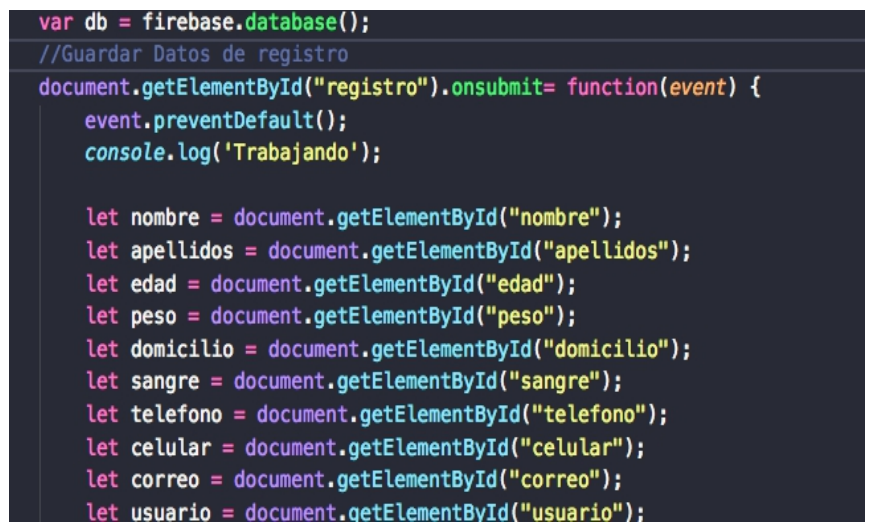

Figure 7. Example of part of the code

In the part of the interaction of the bracelet with the client, a tag language called "HTML5" was used to perform its interface since most users have used a web interface either on mobile devices or on a computer. Based on the HTML content, the information was taken from W3C (1993).

Likewise, it is managed with a framework called Vue.js which is a progressive framework for the construction of the user's interfaces. Vue is also perfectly capable of supplying energy to sophisticated single-page applications when used in combination with modern tools and libraries of support. It is done with the purpose that the interface is reactive. This means that they work in real time without the need to be loading the document every time one works on the interface. The framework was taken from You Vue.js (2014-2017). 
In the part of the programming, the JavaScript language is being used. It is one of the most used programming languages in the technology industry in the case of interaction of hardware (motors, sensors, LEDs, etc.); in this case, it is with the heart rate sensor and programming (W3C, 1999).

Many tests were carried out with the heart rate sensor to have a certainty of the pulsations already investigated and by the doctors specialized in the area of cardiology. This information about cardiac pulsations must have, on an average, one person who went through interviews with the doctors and investigations already done. Work continues to improve the accuracy of the pulsations that a person can have. Figure 8 represents an example of the tests of the final prototype.

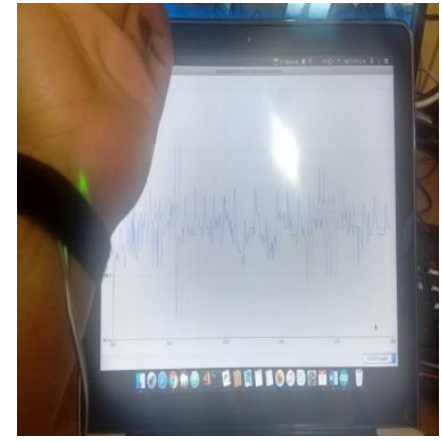

Figure 8. Example of prototype tests

The bracelet will have a database which will allow you to keep track of the different types of pains obtained per day. Likewise, the bracelet will have sample graphs of results obtained from the muscular pains. The use of this bracelet will only be for people who suffer from heart problems.

\section{Implementation}

The final prototype was implemented. Figure 9 represents an example of the digital bracelet.

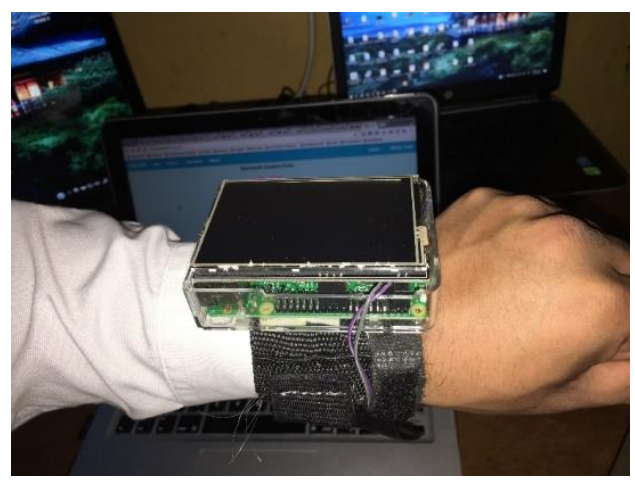

Figure 9. Example of final prototype 
Figure 10 represents the user interface.

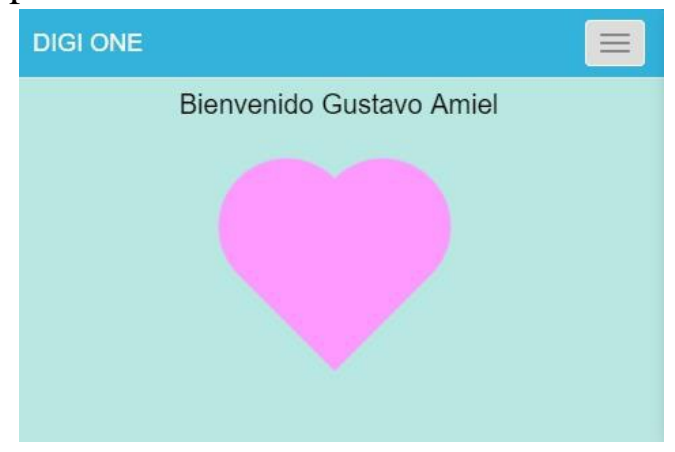

Figure 10. Example of the user's main window

When the product reaches the user's hands, the first thing he will see when he turns on the bracelet will be the next window in which he will have to fill in the information he asks the patient. Figure 11 represents an example of the secondary window.

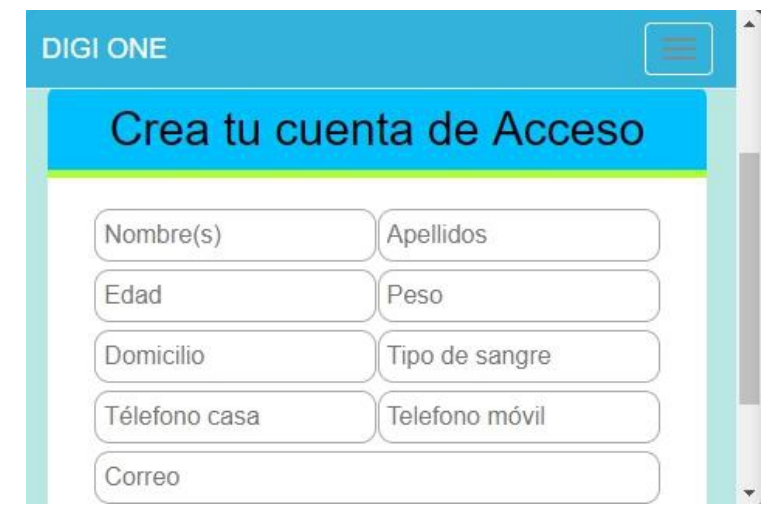

Figure 11. Example of secondary window suffers.

By clicking here, the patient will be able to see the types of pains he

When the patient selects a pain (in this example the headache), the user can click on the start button and start counting the time the user feels discomfort. It won't stop until the user presses the stop button.

The (Send) button will send the start time, the type of pain, and the end time of the discomfort to a database in which these data will be stored. This is because with these data, you can create statistics that will help the doctors to have a better diagnosis. Figure 12 represents an example of the aforementioned. 


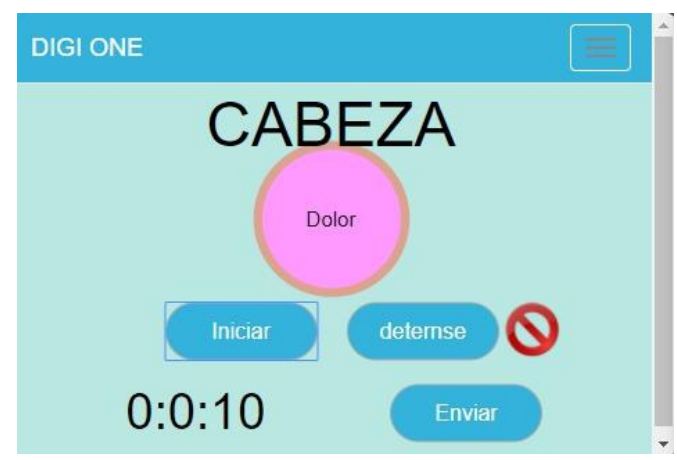

Figure 12. Database example

\section{Results}

With the tests and investigations that were carried out during the creation of the digital wristband, favorable results were obtained, both in the medical specialists as well as in the patients. Thus, it facilitates the process of medical consultation through the use of technology, taking a control of the symptoms of the patient's evolutionary process.

The use of this digital wristband is very pleasant and easy to use, since with just a click on the screen, the patient can add their pain information. With this, the information will be stored in the database that will be connected via local by means of a memory that contains the bracelet. Also, when you have access to the internet, it will go up in the cloud so that the doctor can see the patient's progress.

\section{Acknowledgement}

We wish to acknowledge Román Salgado Maximiliano, Ramirez Miranda María Alejandra, Jaimes Pérez Josué Iván, Martínez Carrillo Eligio, and González Torres Arturo for their advice, guidance, and direction in the course of this study.

\section{References:}

1. Association, A. H. (25 de Enero de 2017). Resumen de estadísticas de 2017.

2. Barrera Chuquiarque, D. E. (2015). Factores de riesgo para enfermedades cardiovasculares según los determinantes de la salud presentes en los choferes de transporte público 2014. E. A. P. de Enfermería. Facultad de Medicina. Universidad Nacional Mayor de San Marcos. Lima, Perú.

3. Banegas. J.R, Villar. F, Graciani. A, Rodríguez Artejo. F. (2006). Epidemiología de las enfermedades cardiovasculares en España. Revista Española de Cardiología. 
4. Eich, B. (2005-2017). MDN web docs. Obtenido de https://developer.mozilla.org/es/docs/Web/JavaScript

5. Eguizábal. P. (2014). La mHealth moverá 4.000 millones de euros en España en 2015. Madrid.

6. Lee, A. (2011). Firebase. Obtenido de Firebase: https://firebase.google.com/

7. Ortega, J. C. (2013). Metodologia Evolutivo.

8. Salud, O. M. (2015). Enfermedades cardiovasculares.

9. Salud, O. P. (s.f.). Organización Mundial De La Salud.

10. Organización Mundial de la Salud. (2013). Estadísticas sanitarias mundiales 2013. Ginebra: OMS.

11. Organisation for economic co-operation and development (2013). Health data 2013. OECD.

12. Tamplin, J. (2011). Firebase. Obtenido de Firebase: https://firebase.google.com/

13. UNED (2017). Introducción: Un poco de historia. Alimentación en las enfermedades: Cardiovasculares. Guía de Alimentación y Salud Cardiovasculares. Facultad de Ciencias. Nutrición y Dietética.UNED.

14. Universia (27 de septiembre de 2010). Enfermedades cardiacas, primera causa de muerte en México.

15. Virtual, E. R. (20 de junio de 2010). Posts Tagged desarrollo evolutivo.

16. W3C (1993). The World's Largest Web Developer Site. Obtenido De The World's Largest Web Developer Site: https://www.w3schools.com/html/

17. W3C (1999-2017). The World's Largest Web Developer Site.

18. You, E. (2014-2017). Vue.js. Obtenido de Vue.js: https://vuejs.org/

19. You, E. (s.f.). Vue.js. Obtenido de Vue.js: https://vuejs.org/v2/guide/\#What-is-Vue-js 